Conclusions Treatment with the single tablet regimen of SOF/ VEL for 12 weeks was highly effective and well tolerated in genotype 1-4 HCV-infected liver transplant recipients with and without cirrhosis.

\section{IDDF2018-ABS-0110 EFFICACY AND SAFETY OF SOFOSBUVIR/ VELPATASVIR PLUS RIBAVIRIN FOR 12 OR 24 WEEKS IN GENOTYPE 1 OR 2 HCV- INFECTED JAPANESE PATIENTS WITH PRIOR TREATMENT FAILURE TO DAA-BASED REGIMENS}

${ }^{1}$ Namiki Izumi*, ${ }^{2}$ Tetsuo Takehara, ${ }^{3}$ Kazuaki Chayama, ${ }^{4}$ Hiroshi Yatsuhashi, ${ }^{5}$ Koichi Takaguchi, ${ }^{6}$ Tatsuya Ide, ${ }^{1}$ Masayuki Kurosaki, ${ }^{7}$ Yoshiyuki Ueno, ${ }^{8}$ Hidenori Toyoda, ${ }^{9}$ Satoru Kakizaki, ${ }^{10}$ Yasuhito Tanaka, ${ }^{3}$ Yoshiiku Kawakami, ${ }^{11}$ Hirayuki Enomoto, ${ }^{12}$ Fusao Ikeda, ${ }^{13} \mathrm{KC}$ Huang, ${ }^{13}$ Shampa De-Oertel, ${ }^{13}$ Brian L McNabb, ${ }^{13}$ Gregory Camus, ${ }^{13}$ John McNally, ${ }^{13}$ Diana M Brainard, ${ }^{13}$ Christina Sze Man Yip, ${ }^{13}$ Hai Cheng Huang, ${ }^{13}$ John

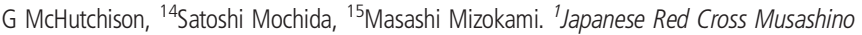
Hospital, Tokyo, Japan; ${ }^{2}$ Osaka University Hospital, Osaka, Japan; ${ }^{3}$ Hiroshima University Hospital, Hiroshima, Japan; ${ }^{4}$ Nagasaki Medical Center, Nagasaki, Japan; ${ }^{5}$ Kagawa Prefectural Central Hospital, Takamatsu, Japan; ${ }^{6}$ Kurume University Hospital, Kurume-shi, Japan; ${ }^{7}$ Yamagata University Hospital, Yamagata, Japan; ${ }^{8}$ Ogaki University Hospital, Ogaki City, Japan; ${ }^{9}$ Gunma University Hospital, Gunma, Japan; ${ }^{10}$ Nagoya City University Hospital, Nagoya, Japan; ${ }^{11}$ Hyogo College of Medicine Hospital, Hyogo, Japan; ${ }^{12}$ Okayama University Hospital, Okayama, Japan; ${ }^{13}$ Gilead Sciences, Inc, Foster City, CA, United States; ${ }^{14}$ Saitama Medical University Hospital, Iruma-gun, Japan; ${ }^{15}$ National Center for Global Health and Medicine, Ichikawa-shi, Japan

\subsection{6/gutjnl-2018-IDDFabstracts.211}

Background There is a growing number of Japanese patients with HCV infection who have failed direct acting antiviral (DAA)-based regimens and currently have no salvage therapies available. This Phase 3 study evaluates the efficacy and safety of sofosbuvir/velpatasvir (SOF/VEL) plus ribavirin (RBV) for 12 or 24 weeks in Japanese patients with genotype (GT) 1 or 2 HCV infection who have been previously treated with DAAs.

Methods Approximately 110 subjects were randomised 1:1 to receive SOF/VEL+RBV for 12 or 24 weeks. Randomization was stratified by GT and presence of cirrhosis. All subjects must have been previously treated with a DAA for at least 4 weeks. Subjects with GT1 HCV infection must have previously been treated with an NS5A inhibitor. The primary efficacy analysis is a comparison of the SVR12 rates for GT1 patients in each of the two treatment groups to a historical control SVR of $50 \%$.

Results Of 117 patients enrolled, 45\% were male, 81\% had GT1 HCV infection, and 33\% had cirrhosis. $84 \%$ had previously been treated with at least 2 different DAAs.86\% of GT1 patients had previously been treated with daclatasvir plus asunaprevir and 91\% of GT2 patients with SOF. Virologic outcomes at post-treatment week 4 are presented in the table below. There were no on-treatment virologic failures. Complete SVR12 and virology data will be presented. Three (3\%) patients discontinued study drugs due to adverse events (AEs). One patient in the 12 week arm discontinued study drugs on Day 4 due to rash (related to study drugs). Two patients in the 24 week arm discontinued study drugs; one on Day 85 due to hepatic angiosarcoma (not related) and one on Day 57 due to depression (related). The two latter patients achieved SVR12. No Grade 3 or 4 AEs were considered related to study drugs (table 1 ).
Conclusions SOF/VEL+RBV has the potential to be a safe, well-tolerated, and effective treatment for Japanese patients with and without cirrhosis who have previously failed DAAbased regimens, a group without currently approved retreatment options. Baseline NS5A RASs did not affect treatment outcome.

Abstract DDF2018-ABS-0110 Table 1 Virologic Outcomes for DAA-Experienced Patients Treated with SOF/VEL+RBV for 12 or 24 Weeks

\begin{tabular}{|c|c|c|c|c|c|c|}
\hline & & 12 Weeks & & & 24 Weeks & \\
\hline & Total & GT1 & GT2 & Total & GT1 & GT2 \\
\hline SVR4,\% (n/N) & $\begin{array}{c}86(49 / \\
57)\end{array}$ & $89(42 / 47)$ & $\begin{array}{c}70(71 \\
10)\end{array}$ & $\begin{array}{c}98(59 / \\
60)\end{array}$ & $98(47 / 48)$ & $\begin{array}{c}100 \\
(12 / 12)\end{array}$ \\
\hline $\begin{array}{l}\text { With BL NS5AVEL- } \\
\text { specific RASs }\end{array}$ & $\begin{array}{c}87(47 / \\
54)\end{array}$ & $89(40 / 45)$ & $\begin{array}{l}78(71 \\
9)\end{array}$ & $\begin{array}{c}100 \\
(52 / 52)\end{array}$ & $\begin{array}{c}100(41 / \\
41)\end{array}$ & $\begin{array}{c}100 \\
(11 / 11)\end{array}$ \\
\hline $\begin{array}{l}\text { Without BL NS5A VEL- } \\
\text { specific RASs }\end{array}$ & $\begin{array}{l}67(21 \\
3)\end{array}$ & $100(2 / 2)$ & $\begin{array}{l}0(0 / \\
1)\end{array}$ & $88(7 / 8)$ & $86(6 / 7)$ & $\begin{array}{l}100(1 / \\
1)\end{array}$ \\
\hline Relapse, $\%(\mathrm{n} / \mathrm{N})$ & $\begin{array}{c}12(71 \\
57)\end{array}$ & $9(4 / 47)$ & $\begin{array}{c}30(3 / \\
10)\end{array}$ & $2(1 / 60)$ & $2(1 / 48)$ & 0 \\
\hline
\end{tabular}

*RAS.resistance-associated substitution

\section{IDDF2018-ABS-0111 SAFETY AND EFFICACY OF TREATMENT WITH ONCE-DAILY LEDIPASVIR/ SOFOSBUVIR ( $90 / 400$ MG) FOR 12 WEEKS IN GENOTYPE 1 HCV-INFECTED PATIENTS WITH SEVERE RENAL IMPAIRMENT}

${ }^{1}$ Eric Lawitz* ${ }^{2}$ Charles S Landis, ${ }^{3}$ Benedict J Maliakkal, ${ }^{4}$ Maurizio Bonacini, ${ }^{5}$ Grisell OrtizLasanta, ${ }^{6}$ Jie Zhang, ${ }^{6}$ Erik Mogalian, ${ }^{6}$ Shampa De-Oertel, ${ }^{6}$ Anu 0 Osinusi, ${ }^{6}$ Diana M Brainard, ${ }^{6}$ John G McHutchison, ${ }^{6}$ Christina Sze Man Yip, ${ }^{6}$ Hai Cheng Huang, ${ }^{7}$ Steven L Flamm, ${ }^{8}$ Stuart C Gordon, ${ }^{9}$ Edward I Gane. ${ }^{1}$ University of Texas Health San Antonio, Texas Liver Institute, USA; ${ }^{2}$ University of Washington Medical Center, Seattle, WA, USA; ${ }^{3}$ University of Tennessee, Memphis, TN, USA; ${ }^{4}$ Mission Gastroenterology and Hepatology, UCSF, San Francisco, CA, USA; ${ }^{5}$ Fundacion de Investigacion de Diego, San Juan, PR, USA; ${ }^{6}$ Gilead Sciences, Inc, Foster City, CA, USA; ${ }^{7}$ Division of Gastroenterology and Hepatology, Northwestern University, Chicago, IL, USA; ${ }^{8}$ Henry Ford Health System, Detroit, MI, USA ${ }^{9}$ Auckland Clinical Studies, Auckland, New Zealand

\subsection{6/gutjnl-2018-IDDFabstracts.212}

Background Despite higher concentrations of the primary circulating sofosbuvir (SOF) metabolite, GS-331007, in patients with severe renal impairment (RI), retrospective case series and claims database analyses have suggested substantial use of ledipasvir (LDV)/SOF in this population with no untoward effects described. The current study evaluated the safety, efficacy, and pharmacokinetics (PK) of LDV/SOF (90/400 mg) once-daily for 12 weeks in patients with genotype (GT) 1 $\mathrm{HCV}$-infection and severe RI.

Methods Treatment naive or experienced patients with or without compensated cirrhosis and creatinine clearance (CLcr). Results Of the 18 patients, the majority were male (67\%), 10 (56\%) were African-American, 8 (44\%) had $\mathrm{BMI} \geq 30 \mathrm{~kg} / \mathrm{m} 2$ and mean (range) CLcr at baseline was 24.9 (9.0-39.6) mL/ min. In terms of liver disease characteristics, all 18 had GT1 HCV infection (14 GT1a and 4 GT1b), 14 (78\%) were treatment naive, and $2(11 \%)$ had cirrhosis. All patients completed 12 weeks of LDV/SOF treatment. There were no early discontinuations nor any on-treatment virologic failures. The SVR12 rate is $100 \%(18 / 18)$. Plasma concentrations of the terminal 
SOF metabolite GS-3310007 were approximately 6 fold higher than in the LDV/SOF Phase 3 trials. SOF and LDV concentrations were similar to those with normal, mild or moderate RI. The most common adverse events (AEs) were fatigue (22\%), headache (22\%), and hyperkalemia (22\%). Six serious AEs were reported among 4 patients (22\%), including 2 renal events; no SAEs related to study drugs. There were no treatment-related cardiac AEs, including bradycardia, and no meaningful changes in QTc intervals or other parameters.

Conclusions Treatment with LDV/SOF (90/400 mg) for 12 weeks in genotype 1 patients with and without cirrhosis and severe renal impairment resulted in 100\% SVR12 rate. The regimen was safe and well-tolerated with no treatment discontinuations and no treatment-related SAEs. (no table selected) (No Image Selected) Co-Author Disclosure Status.

\section{IDDF2018-ABS-0112 SAFETY AND EFFICACY OF SOFOSBUVIR/ VELPATASVIR IN A GENOTYPE 1-6 HCV INFECTED POPULATION FROM SINGAPORE, MALAYSIA, THAILAND, AND VIETNAM: RESULTS FROM A PHASE 3, CLINICAL TRIAL}

${ }^{1}$ Seng Gee Lim*, ${ }^{2}$ Rosmawati Mohamed, ${ }^{3}$ Phuong Le, ${ }^{4}$ Hoi Poh Tee, ${ }^{5}$ Brian McNabb, ${ }^{5}$ Sofia Lu, Julia Lu, ${ }^{5}$ John McNally, ${ }^{5}$ Diana M Brainard, ${ }^{5} \mathrm{G}$ Mani Subramanian, ${ }^{5}$ Christina Sze Man Yip, ${ }^{5}$ Hai Cheng Huang, ${ }^{6}$ Chee Kiat Tan, ${ }^{7}$ Tawesak Tanwandee, ${ }^{8}$ Kinh Nguyen Van, ${ }^{9}$ Teerha Piratvisuth. 'Division of Gastroenterology and Hepatology, National University Hospital, Singapore; ${ }^{2}$ Department of Medicine, Faculty of Medicine, University of Malaya, Kuala Lumpur, Malaysia; ${ }^{3}$ People's Hospital 115, Ho Chi Minh City, Vietnam; ${ }^{4}$ Gastroenterology Unit, Medical Department, Hospital Tengku Ampuan Afzan, Jalan Tanah Putih, Kuantan, Pahang, Malaysia; ${ }^{5}$ Gilead Science Inc., Foster City, CA, USA; ${ }^{6}$ Department of Gastroenterology and Hepatology, Singapore General Hospital, Singapore; 'Division of Gastroenterology, Department of Medicine, Siriraj Hospital, Mahidol University, Bangkok, Thailand; ${ }^{8}$ National Hospital for Tropical Diseases, Hanoi, Vietnam; ${ }^{9}$ NKC Institute of Gastroenterology and Hepatology, Faculty of Medicine, Prince of Songkla University, Hat Yai, Songkhla, Thailand

\subsection{6/gutjnl-2018-IDDFabstracts.213}

Background The prevalence of hepatitis C virus (HCV) in Singapore, Malaysia, Thailand, and Vietnam, ranges from 1\%$3 \%$. Because of the heterogeneity of genotypes (GTs) in these countries (primarily GT1, 2, 3, and 6), there is a critical need for a pangenotypic, all oral regimen to address the burden of HCV infection. This study evaluated the efficacy and safety of SOF/VEL for 12 weeks in patients with chronic GT1-6 HCV infection.

Methods Treatment experienced and treatment naive patients with chronic GT1-6 HCV infection with no cirrhosis or with compensated cirrhosis were eligible to enrol in a single-arm, open-label trial to receive a fixed dosed combination of SOF/ VEL 400/100 mg daily for 12 weeks. Patients were recruited from 13 sites in Singapore, Malaysia, Thailand, and Vietnam. The primary efficacy endpoint was SVR12 using the CAP/ CTM HCV 2.0 assay $(\mathrm{LLOQ}=15 \mathrm{IU} / \mathrm{mL})$, and the primary safety endpoint was adverse events (AEs) leading to SOF/VEL discontinuation.

Results A total of 111 patients were enrolled and treated. Of these, 51\% were male, $14 \%$ had compensated cirrhosis, $18 \%$ were treatment-experienced, $82 \%$ had IL28B CC genotype, 20\% had GT1a, 23\% GT1b, 3\% GT2, 23\% GT3, and 31\% GT6 HCV infection. The overall SVR12 rate was 97\% (108/ 111) GT specific SVR12 rates are presented in the Table
(IDDF2018-ABS-0112 Table 1). All 3 patients who did not achieve SVR experienced a virologic relapse. All 15 cirrhotic patients, including 8 with GT3 HCV infection achieved SVR12. Virology data will be presented. There were no discontinuations due to AEs. There were 50 patients (45\%) who experienced any adverse events (AEs), with no AEs occurring in $>10 \%$ of patients. No serious or severe AEs were assessed by the investigator as related to study drug and there were no deaths.

\begin{tabular}{|c|c|c|c|c|c|c|c|c|}
\hline \multirow[t]{2}{*}{$\mathrm{N}(\%)$} & Total & GT1a & GT1b & GT1 & GT2 & GT3 & GT6 & Indeterminate ${ }^{\mathrm{a}}$ \\
\hline & $\mathrm{n}=111$ & $n=22$ & $n=25$ & $\begin{array}{l}\text { (total) } \\
n=47\end{array}$ & $n=3$ & $\mathrm{n}=25$ & $n=34$ & $n=2$ \\
\hline SVR12 & $\begin{array}{c}108 / 111 \\
(97)\end{array}$ & $\begin{array}{l}22 / 22 \\
(100)\end{array}$ & $\begin{array}{l}25 / 25 \\
(100)\end{array}$ & $\begin{array}{l}47 / 47 \\
(100)\end{array}$ & $\begin{array}{c}3 / 3 \\
(100)\end{array}$ & $\begin{array}{c}23 / 25 \\
(92)\end{array}$ & $\begin{array}{c}33 / 34 \\
(97)\end{array}$ & $2 / 2(100)$ \\
\hline
\end{tabular}

Conclusions Treatment with the single tablet, pangenotypic regimen of SOF/VEL for 12 weeks was highly effective and well tolerated in a GT 1,2,3, and $6 \mathrm{HCV}$ infected population with and without cirrhosis from Singapore, Malaysia, Thailand, and Vietnam. *Seng Gee Lim is the presenter of the abstract.

\section{IDDF2018-ABS-0114 SOF/VEL/VOX RESULTS IN HIGH SVR12 RATES WHEN ADMINISTERED FOR 12 WEEKS IN DAA-EXPERIENCED PATIENTS OR FOR 8 WEEKS IN DAA-NAIVE PATIENTS: AN INTEGRATED ANALYSIS OF THE POLARIS-1, POLARIS-2, POLARIS-3 AND POLARIS-4 STUDIES}

${ }^{1}$ Stuart K Roberts* ${ }^{2}$ Curtis L Cooper, ${ }^{3}$ Eric Lawitz, ${ }^{4} \mathrm{~K}$ Rajender Reddy, ${ }^{5}$ Alex J Thompson, ${ }^{6}$ Stefan Zeuzem, ${ }^{7}$ Ira M Jacobson, ${ }^{8}$ Peter Ruane, ${ }^{9}$ Robert H Hyland, ${ }^{9}$ Luisa M Stamm, ${ }^{9}$ Lingling Han, ${ }^{9}$ Diana M Brainard, ${ }^{9}$ Christina SM Yip, ${ }^{9} \mathrm{HC}$ Huang, ${ }^{10}$ Norbert Brau, ${ }^{11}$ Tarik Asselah, ${ }^{12}$ Bernard E Willems, ${ }^{13}$ Steven Flamm, ${ }^{14}$ Marc Bourliere, ${ }^{15} \mathrm{Graham}$ R Foster, ${ }^{16}$ Edward J Gane, ${ }^{17}$ Michael Manns, ${ }^{18}$ Stuart C Gordon, ${ }^{19}$ Kris Kowdley. ${ }^{1}$ Alfred Hospital, Melbourne, Australia; ${ }^{2}$ Ottawa Hospital Research Institute, Ottawa, Canada; ${ }^{3}$ Texas Liver Institute, University of Texas Health Science Center, San Antonio, USA; ${ }^{4}$ University of Pennsylvania, Philadelphia, USA; ${ }^{5}$ Department of Gastroenterology, St. Vincent's Hospital, Melbourne, Australia; ${ }^{6} J$ Johann Wolfgang Goethe University Medical Center, Frankfurt, Germany; ' Department of Medicine, Mount Sinai Beth Israel, New York, USA; ${ }^{8}$ Ruane Medical and Liver Health Institute, Los Angeles, USA; ${ }^{9}$ Gilead Sciences, Inc., Foster City, USA; ${ }^{10}$ Mount Sinai School of Medicine, New York, USA; ${ }^{11}$ Service $d^{\prime}$ Hepatologie, Hopital Beaujon, Universite Paris Diderot, Clichy, France; ${ }^{12}$ Northwestern University, Chicago, USA; ${ }^{13}$ Hospital Saint Joseph, Marseille, France; ${ }^{14}$ Royal London Hospital, London, UK; ${ }^{15}$ Auckland Clinical Studies, Auckland, New Zealand; ${ }^{16}$ Department of Gastroenterology, Hepatology and Endocrinology, Hannover Medical School, Hannover, Germany; ${ }^{17}$ Henry Ford Health System, Detroit, USA; ${ }^{18}$ Swedish Medical Center, Seattle, USA; ${ }^{19}$ Swedish Medical Center, Seattle, United States

\subsection{6/gutjnl-2018-IDDFabstracts.214}

Background The once-daily fixed-dose combination tablet of sofosbuvir/velpatasvir/voxilaprevir(SOF/VEL/VOX) was evaluated for the treatment of genotype 1-6 HCV infection in four Phase 3 studies in direct-acting antiviral (DAA)-experienced (POLARIS-1 and POLARIS-4) and DAA-naive 